ARTIGO

Recebido em:

$02 / 12 / 2014$

Aceito em:

01/04/2015

\title{
A geração e o gerenciamento de referências bibliográficas com o Mecanismo Online para Referências - MORE
}

\author{
The generation and management of references with the Online \\ Mechanism for References - MORE
}

\author{
Proxério Manoel Felisberto \\ Universidade Federal de Santa Catarina \\ proxerio.felisberto@posgrad.ufsc.br \\ Roderval Marcelino \\ Universidade Federal de Santa Catarina \\ roderval.marcelino@ufsc.br \\ Maria Bernardete Martins Alves \\ Universidade Federal de Santa Catarina \\ m.alves@ufsc.br \\ João Bosco da Mota Alves \\ Universidade Federal de Santa Catarina \\ jbosco@inf.ufsc.br \\ Juarez Bento da Silva \\ Universidade Federal de Santa Catarina \\ juarez.silva@ufsc.br \\ Vilson Gruber \\ Universidade Federal de Santa Catarina \\ vilson.gruber@ufsc.br
}

\section{Resumo}

A produção científica e a elaboração de trabalhos acadêmicos revestem-se de formalidades próprias. Destas formalidades busca-se, neste trabalho, abordar as que se referem à forma de concessão dos méritos aos autores das obras utilizadas no embasamento teórico, através das citações e referências bibliográficas. 0 que se pretende é o auxilio aos usuários de biblioteca na geração e gerenciamento de referências fazendo uso de uma ferramenta web desenvolvida para esta finalidade. Vários são os aplicativos, sejam eles desktop e/ou disponibilizados através da $w e b$, que poderiam suprir esta necessidade. No entanto, alguns requerem a aquisição de algum tipo de licença onerosa para desfrutar de todas as suas possibilidades. Outros possuem a versão gratuita, todavia, são muito limitadas e por vezes não contemplam a geração de referências nos moldes especificados pela ABNT. Existem outras que não disponibilizam a funcionalidade de armazenamento das referências geradas para serem utilizadas em momento posterior. No intuito de suprir esta lacuna foi desenvolvido e disponibilizado ao público em geral, através da web, o Mecanismo Online para Referências (MORE), no ano de 2006. Entretanto, o rápido avanço tecnológico aliado ao aumento do número de usuários do sistema contribuíram para a necessidade de atualização do mesmo, realizada recentemente. Ressalta-se que todo o trabalho foi desenvolvido, exclusivamente, com o uso de tecnologias gratuitas e popularmente consagradas. Inicialmente, buscou-se identificar quais são as principais ferramentas geradoras e/ou gerenciadoras de referências bibliográficas disponíveis para uso e quais tecnologias gratuitas poderiam ser utilizadas na construção de sistemas web interativos. Na sequência descreve-se, de forma sucinta o processo de reengenharia a que o MORE foi submetido, sua nova estrutura, novos requisitos implementados com sua remodelagem e ampliação do seu portfólio de funcionalidades. Finalizando, faz-se uma síntese dos resultados alcançados atualmente em contrapartida aos resultados anteriores à sua atualização.

v. 20, n. 42,2015 p. $79-92$

ISSN 1518-2924

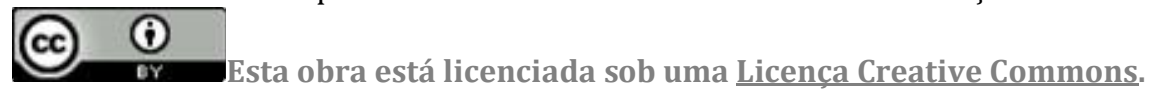


Palavras-Chave: Referências bibliográficas. Sistema web. Coleções de referências. Tecnologias da informação e comunicação.

\begin{abstract}
The scientific production and the development of academic papers have their own formalities. In this paper, is sought seek from these formalities for the ones that refers to the way of granting merits to the authors of the works used in the theoretical basis, through quotations and references. The goal is to help users of libraries to generate and manage references using a web tool developed for this purpose. There are many applications, in desktop and web platforms, that could be used to do this task. However, some of them require the payment of an expensive license to be fully functional. Others offers free versions, but they are very limited and often do not generate references specified by ABNT. There are others that do not store the generate references for later use. In order to fill this gap, the Online Mechanism for References (MORE) was developed and made available to the general public in the web. Even so, the fast technological advances combined with a high number of users demanded an update to the application, done recently. It is important to state that all the work was developed exclusively with proven and free to use technologies. Initially, sought up identified the main tools available to generate and manage references and which free technologies could be used to build interactive web applications. This paper briefly describes the reengineering process that MORE was submitted, its new structure, new requirements met and its expanded portfolio of features. Finally, the results achieved after the reengineering are compared to indicators of its previous version.

Keywords: References. Web system. Collection references. Information and communication technologies.
\end{abstract}

\title{
1 INTRODUÇÃO
}

O paradigma referente ao processo de construção do conhecimento sofreu algumas alterações ao longo da história da humanidade. Foram sucessivos avanços até chegar-se aos rigorosos métodos atuais da pesquisa e do desenvolvimento científico, amplamente reconhecidos por toda e comunidade envolvida neste contexto. Dentre as técnicas consagradas e amplamente utilizadas destaca-se a utilização de trabalhos já realizados, de preferência por autores reconhecidos no meio científico ou que constem de publicações renomadas na área do saber objeto da pesquisa, para fundamentar ou realizar questionamentos relativos ao tema abordado. Esta técnica requer a concessão dos méritos ao autor, normalmente na forma de citações e referenciamento bibliográfico.

Constata-se a existência de vários órgãos, conhecidos mundialmente, que normatizam, segundo suas diretrizes, a forma como essas referências devem ser utilizadas. Observa-se, também, que os países possuem algum tipo de órgão, associação ou agremiação, responsável por este trabalho no âmbito nacional.

O Brasil, atualmente, conta a Associação Brasileira de Normas Técnicas (ABNT) cuja principal finalidade é a normalização técnica em todo o território nacional, abrangendo as mais diversas áreas do conhecimento (ABNT, 2014).

Pesquisadores, professores, alunos e usuários de bibliotecas geralmente têm despendido muito tempo para tomar conhecimento das normas que regem o assunto, bem como, para elaborar sua lista de referências bibliográficas utilizadas no desenvolvimento do trabalho em curso. A fim de minimizar o problema e colaborar com estas pessoas neste 
tipo de empreendimento, surgiu, em 2006, o Mecanismo Online para Referências (MORE) que oferece, gratuitamente, ao público em geral a possibilidade, através da web, de geração e gerenciamento de referências bibliográficas segundo as normas da ABNT, mantendo-as armazenadas em seu servidor.

Estes usuários dispõem de algumas ferramentas que os auxiliam na elaboração e no gerenciamento de suas referências bibliográficas, conforme explorado na próxima seção, todavia o MORE visa auxiliar àqueles que buscam uma ferramenta gratuita, que faça o armazenamento e o gerenciamento de suas referências e que ofereça este serviço segundo as normas da ABNT.

Os avanços tecnológicos verificados na última década, bem como a popularização da internet e as políticas públicas de inclusão digital, aliado ao aumento do número de usuários do aplicativo, exigem que os sistemas que operam neste ambiente apresentem elevado grau de escalabilidade, robustez, confiabilidade, confidencialidade, rapidez e interatividade, bem como características ergonômicas e de acessibilidade. Neste contexto, verificou-se a necessidade de atualização do MORE para que sua estrutura e suas funcionalidades evidenciem as características desejadas nas grandes aplicações web (LOUDON, 2010). Tal atualização foi conduzida com a utilização de tecnologias gratuitas o que possibilita a disseminação dos paradigmas atuais de desenvolvimento para a web, tais como: HTML, CSS, PHP e Java Script, entre outros.

Para alcançar o objetivo de atualização do MORE desenvolveu-se a pesquisa segundo os preceitos da Metodologia da Experimentação, que para Cervo, Bervian e Silva (2007) "é o conjunto de processos utilizados para verificar as hipóteses [...] e o princípio geral no qual se fundamentam as técnicas da experimentação é o do determinismo", ou seja, "nas mesmas circunstâncias, as mesmas causas produzem os mesmos efeitos".

No planejamento e execução do projeto de atualização do sistema propriamente dito foram utilizados métodos ágeis, mais precisamente o SCRUM (Software Creation Revolutionary Unified Modelling). Esta metodologia baseia o desenvolvimento do software nos princípios ágeis e no Manifesto Ágil, ocorrendo de forma iterativa, através de uma série de sprints. Um sprint pode ser entendido como um lapso de tempo, com duração entre duas e quatro semanas, que são utilizadas para implementar alguma funcionalidade no software e entregá-lo, ainda que incompleto (BLANKENSHIP; BUSSA; MILLETT, 2012).

A Figura 1 mostra graficamente as etapas do processo de construção de um software utilizando-se a metodologia SCRUM. 
Figura 1: 0 processo SCRUM.

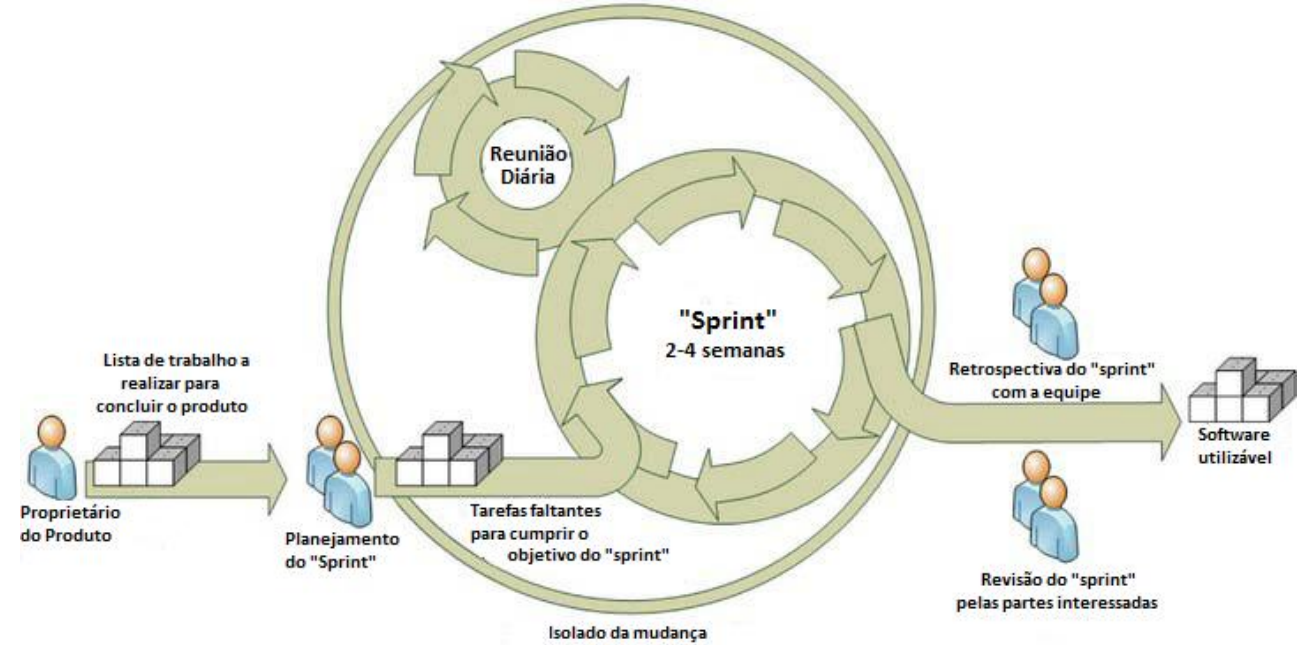

Fonte: adaptada de Blankenship, Bussa e Millett (2012).

O MORE conta atualmente com mais de 115.000 usuários cadastrados e sua modernização visou atender um público bem específico, ou seja, aquele que necessita uma ferramenta de gerenciamento de referências bibliográficas gratuita, em português, que gere suas referências de acordo com a NBR 6023 (2002), que não está disposto a pagar pelo uso de um gerenciador proprietário e que possa manter suas referências armazenadas e disponíveis na web para serem acessadas a qualquer momento e de qualquer lugar (ALVES, MENDES; ALVES, 2007).

\section{GERENCIADORES DE REFERÊNCIAS BIBLIOGRÁFICAS}

Esta seção tem por escopo fazer um breve apanhado sobre os principais gerenciadores de referências bibliográficas disponíveis aos usuários de bibliotecas, no entanto, alguns são proprietários e requerem a aquisição de algum tipo de licença para a sua plena utilização.

Um gerenciador de referências interessante é o JABREF Reference Manager, uma vez que é gratuito, desenvolvido em Java, cuja execução se dá através da Máquina Virtual Java 1.5 ou superior, o que lhe confere a portabilidade para várias plataformas. Segundo SOURCEFORGE.NET (2014), apesar de exportar suas referências para vários tipos de arquivos, inclusive para o editor de textos Microsoft Word $^{\mathrm{TM}}$, sua característica principal é usar nativamente o formato de arquivo BibTex, o mesmo utilizado pelo LATEX para gerenciar as referências bibliográficas. Possibilita ao usuário a personalização dos tipos de referências, inclusive a inclusão, exclusão ou alteração de campos, sejam eles obrigatórios, opcionais ou de uso geral. A base de dados gerada no JabRef pode ser acessada diretamente pelo LATEX bastando apenas informar o caminho do arquivo BibTex.

Os assinantes da plataforma ISI Web of Knowledge (Web of Science) contam com uma ferramenta desenvolvida pela Thomson Reuters, o EndNote $\boldsymbol{W e b}^{\circledR}$. Segundo Pestana e Funaro (2009) trata-se de "um software de gestão pessoal que auxilia o aluno de graduação, pós-graduação, docente e pesquisador na elaboração de artigos de pesquisa". E para Estorniolo Filho (2013) "permite armazenar e organizar as referências encontradas nas buscas em bases de dados, possibilitando a inclusão automática de citações e 
referências quando da elaboração de um texto". É o gerenciador de referências preferido dos usuários do editor de textos Microsoft Word $^{\mathrm{TM}}$. Na realidade o $\boldsymbol{E n d N o t e}^{\circledR}$ dispõe de três formas de utilização. 0 mais complexo e que apresenta maior número de funcionalidades é o EndNote ${ }^{\circledR}$ para desktop. Temos ainda o EndNote $\boldsymbol{W e b}^{\circledR}$ para assinantes e o EndNote Web ${ }^{\circledR}$ versão livre. Todavia, com pouquíssimos recursos, se comparado aos demais.

O Facilis é uma ferramenta web gratuita que possibilita a geração de referências, conforme os padrões estabelecidos pela ABNT, através da inserção manual dos dados e o idioma utilizado é o português brasileiro. Está disponível no endereço eletrônico <http://facilis.uesb.br $>$. Se propõe a gerar todos os tipos de referências constantes na NBR 6023 (2002), no entanto, não oferece a possibilidade de gerenciamento destas através de algum dispositivo de armazenamento e não faz nenhuma alusão à NBR 10520 (2002), ou seja, não auxilia seu usuário no uso de citações.

Na sequência explora-se algumas características do ProCite ${ }^{\circledR}$, que é uma ferramenta proprietária de gerenciamento de documentos e referências bibliográficas do Institute for Scientific Information (ISI). Útil para organizar referências e criar bibliografias automaticamente a partir de um processador de textos. Permite construir bases de dados com as referências bibliográficas recuperadas através das pesquisas realizadas em qualquer suporte (UNIVERSIDAD DE SEVILLA, 2006). Trata-se de um sistema de banco de dados de propósito específico uma vez que não necessita nenhuma programação para gerenciar grandes bases de dados com registros de tamanhos variáveis. Trabalha com o conceito de workforms que oferece ao usuário uma espécie de modelo contendo as entradas e as saídas já formatadas para os mais diversos tipos de documentos, tais como: livros, monografias, artigos de periódicos, fotografias, filmes, patentes e mapas, entre outros.

Uma das ferramentas proprietárias da Thomson Reuters para gerenciamento de referências que se destaca é o Reference Manager $^{\circledR}$, uma vez que importa referências bibliográficas de bases de dados remotas; permite organizar com facilidade uma coleção pessoal de referências e sua publicação na web ou em uma intranet; disponibiliza o plugin Cite While You Write para utilização no MS Word $^{\mathrm{TM}}$ ou Corel ${ }^{\circledR}$ WordPerfect; permite o compartilhamento em rede da coleção de referências.

No âmbito do THE LANDMARK PROJECT (2014) desenvolveu-se o Citation Machine que tem por objetivo auxiliar estudantes e pesquisadores profissionais na elaboração de referências bibliográficas, fazendo com que esta tarefa se torne um procedimento fácil. Oferece a possibilidade de utilização nos formatos MLA, APA, TURABIAN e CHICAGO. O sistema é gratuito e interativo. Encontra-se disponível na web e não suporta a possibilidade de customização para outro formato além dos 4 (quatro) já mencionados.

O EasyBib, da PALOMAR COLLEGE LIBRARY (2014), é uma ferramenta online que ajuda a criar citações nos estilos MLA, APA e Chicago/Turabian. Operacionaliza a criação de uma lista de obras referenciadas. Assim, em muitos casos, EasyBib pode criar uma referência automaticamente (AutoCITE) se reconhecer o livro, site, artigo de revista, etc, que está sendo citado. 


\section{TECNOLOGIAS GRATUITAS UTILIZADAS NA ELABORAÇÃO DE SISTEMAS WEB}

Busca-se, nesta seção, fazer uma descrição sucinta de algumas definições e características encontradas nas várias tecnologias empregadas, atualmente, no desenvolvimento de sistemas para a web. Serão abordadas apenas tecnologias disponibilizadas gratuitamente, uma vez que as proprietárias não se coadunam com o escopo deste trabalho.

\subsection{Ambientes de Desenvolvimento Integrado (IDE)}

Também conhecidos como IDE, do inglês Interface Development Environment, os ambientes de desenvolvimento integrado fazem parte de uma família de aplicativos voltados ao desenvolvimento de softwares. Sua principal finalidade é tornar mais fácil, elegante, robusto e ágil o trabalho dos desenvolvedores, com significativa redução no tempo gasto no processo produtivo de softwares.

Como sugere sua designação, um bom ambiente de desenvolvimento integrado é formado por várias ferramentas que trabalham interligadas a fim de acelerar o processo produtivo e melhorar consideravelmente a qualidade do software produzido. As ferramentas básicas que compõem uma IDE são: um editor de textos (para o código-fonte), uma ferramenta de apoio à gestão de banco de dados, um compilador/interpretador, um depurador e um sistema de controle de versão (TONU, 2012).

O NetBeans é um ambiente de desenvolvimento integrado (IDE), opensource, escrito totalmente em Java. É uma IDE com grande aceitação na comunidade de desenvolvedores de software, desenvolvida pela Sun, e que ao longo dos anos vem se destacando cada vez mais por sua completude, gratuidade e ao mesmo tempo multiplataforma (GONÇALVES, 2006). Vale ressaltar que o NetBeans não se limita à linguagem de programação Java, estando em conformidade com várias outras linguagens. Atentos à crescente demanda do mercado, a partir de sua versão 6.5, que foi lançada em novembro de 2008, incorporou recursos para desenvolvimento em PHP e, atualmente, já é bem popular na comunidade PHP.

No entendimento de Böck (2012), é válido o uso do Netbeans, não importando se o aplicativo a ser desenvolvido seja muito pequeno ou muito grande, em virtude das suas funcionalidades, as quais permitem soluções simples para os problemas e desafios encontrados no percurso do processo de desenvolvimento o que contribui para manutenção de um elevado grau de satisfação de seus usuários.

O projeto Eclipse foi iniciado na IBM que desenvolveu a primeira versão do produto e doou-o como software livre para a comunidade. 0 gasto inicial da IBM no produto foi de mais de 40 milhões de dólares (HOLZNER, 2004). É uma IDE desenvolvida em Java, seguindo o modelo open source de desenvolvimento de software. Suas funcionalidades são baseadas em plugins, que podem ser configuradas de acordo com as necessidades do usuário, de modo simples e ágil. Atenta às tendências da comunidade desenvolvedores de softwares, a Fundação Eclipse lançou o projeto Eclipse PHP Development Tools (PDT), o qual teve excelente aceitação, uma vez que é um dos projetos mais baixados dos repositórios onde se encontra disponível. Sua distribuição padrão conta com suporte às linguagens de script PHP e 
Javascript, sugestões para autocompletamento de código e depuração (local e remota) do código.

O Aptana Studio é uma ferramenta de desenvolvimento profissional para a web, além de ser open source. Possibilita o desenvolvimento e teste da aplicação web completa, usando um único ambiente. Oferece suporte para as mais recentes especificações tecnológicas dos navegadores, tais como: HTML5, CSS3, JavaScript, Ruby on Rails, PHP e Python. Auxilia na criação de HTML, CSS, Java Script, PHP e Ruby.

\subsection{Frameworks}

0 código-fonte do sistema está baseado em dois frameworks (conjunto de ferramentas), um PHP (CodeIgniter) e um CSS (Zurb Foundation).

o Codelgniter é um framework de desenvolvimento de aplicações para desenvolvedores de sítios web usando PHP. Seu objetivo é permitir o desenvolvimento de projetos muito mais rápido do que se tivesse que escrever o código do zero, provendo um vasto conjunto de bibliotecas para tarefas comuns, bem como uma interface simples e uma estrutura lógica para acesso a essas bibliotecas. Upton (2007) afirma ser o Codelgniter uma ferramenta que ajuda os programadores PHP no desenvolvimento de um trabalho melhor, simplificando a leitura e a atualização dos scripts, reduzindo a quantidade de código escrito, estruturando o software em camadas (utiliza o padrão MVC), disciplinando o código e tornando-o mais robusto.

O Zurb Foundation é um framework CSS que facilita sobremaneira a definição do design de uma página, além de engrossar as fileiras dos aplicativos open source. Sua concepção está baseada em um layout de grade flexível de 12 colunas que pode ser definido para um tamanho arbitrário até que se alcance a largura máxima da linha, que também é facilmente aninhada, possibilitando a construção de layouts complexos sem a criação de uma série de elementos personalizados. Dispõe de dezenas de estilos para facilitar a criação rápida de protótipos de um site, vários tamanhos de botões e estilos, guias, elementos de formulário personalizados, caixas de diálogo modais, sliders de imagens e muito mais.

\subsection{Linguagens de marcação, de script e de programação}

Utiliza-se neste projeto as linguagens mais indicadas para a construção de aplicativos web dinâmicos, tais como: PHP, HTML, CSS e JavaScript.

PHP (originalmente significava Personal Home Page, porém de acordo com a convenção para atribuição de nomes recursiva passou a significar PHP Hypertext Preprocessor) é uma linguagem de script open source, de uso geral, muito utilizada e especialmente guarnecida para o desenvolvimento de aplicações web embutida dentro do HTML. Conforme nos ensina Gilmore (2011) verifica-se que as características da linguagem tendem a cair em 4 (quatro) categorias chave: praticidade, poder, possibilidade e preço (é disponibilizado gratuitamente).

HTML é um acrônimo para HyperText Markup Language, cuja tradução para o português é Linguagem para Marcação de Hipertexto. No contexto deste trabalho, hipertexto pode ser entendido como todo o conteúdo inserido em um documento para a web e tem como principal característica a possibilidade de interligação a outros documentos lá existentes. Esta ligação 
é viabilizada através dos enlaces existentes nas páginas web. Atualmente podemos contar com a HTML 5 que, segundo Vaughan-Nichols (2010), "vai apoiar as mais recentes tecnologias móveis, como os serviços de geolocalização, bem como gráficos vetoriais escaláveis (SVG $\left.{ }^{1}\right)^{\prime \prime}$.

A linguagem Java Script nasceu da necessidade de prover interatividade às páginas eletrônicas, uma vez que a HTML não dispunha de elementos capazes de disponibilizar tal funcionalidade. Uma parceria da Netscape com a Sun Microsystems possibilitou seu lançamento em 1995. Silva (2010) ensina que "JavaScript é uma linguagem desenvolvida para rodar no lado cliente, isto é, a interpretação e o funcionamento da linguagem dependem de funcionalidades hospedadas no navegador do usuário". Esta possibilidade é fornecida pelo interpretador Java Script hospedado nesse navegador.

Cascading Style Sheets (CSS), cuja tradução é Folhas de Estilo em Cascata, também são definidas e padronizadas pelo W3C e têm por finalidade devolver à HTML/XML o propósito inicial da linguagem de que era a de marcação e estruturação de conteúdos. Assim, cabe às CSS todas as funções de apresentação de um documento, sendo esta a sua finalidade maior. Daí advém a consagrada frase que resume a dobradinha CSS + HTML: "HTML para estruturar e CSS para apresentar" (SILVA, 2012).

\subsection{SGBDR MySQL}

O Sistema de Gerenciamento de Banco de Dados Relacional (SGBDR) MySQL "é um servidor de banco de dados relacional que se aproxima cada vez mais das facilidades encontradas em produtos proprietários competitivos a cada versão" (GILMORE, 2011).

A popularidade do SGBDR MySQL pode ser atribuída à sua gratuidade para aplicações não comerciais e às seguintes características por ele apresentadas: flexibilidade, capacidade, recursos de SQL a nível de empresa, indexação e busca full-text, query caching, replicação e segurança.

\section{REENGENHARIA DO MORE}

Reengenharia de software, segundo Serra (2009), “é considerada uma modernização de software, ou seja, uma abordagem disciplinada para migrar softwares legados em softwares evolutivos"; e o entendimento de Sommerville (2011) é no sentido de aplicá-la para que se alcance uma "melhoria da estrutura e inteligibilidade" dos sistemas legados. Sistemas legados são aqueles que estão em produção há alguns anos e que, apesar de possuírem um alto valor de negócio, sua manutenção tornou-se muito complexa e dispendiosa.

0 modelo de processo de reengenharia adotado para este trabalho é o sugerido por Sommerville (2011) o qual divide-se em cinco fases: tradução do código-fonte, engenharia reversa, melhoria da estrutura do programa, modularização do programa e reengenharia de dados. Salienta-se que o MORE encontrava-se em execução no servidor do RExLab/UFSC, em Araranguá/SC, e que sua construção anterior foi apoiada na IDE Netbeans, fazendo uso, no lado servidor, da linguagem Java Server Pages (JSP) e, no lado

\footnotetext{
${ }^{1}$ SVG é um formato de arquivo baseado em XML aberto, capaz de produzir gráficos compactos e
} de alta qualidade. 
cliente, Java Script para adicionar dinamicidade às páginas web. A conformação das interfaces apresentadas aos usuários foi baseada em folhas de estilo em cascata (CSS) e a estrutura das informações em HTML. O SGBDR MySQL foi utilizado para a construção e o gerenciamento da base de dados. Todas essas tecnologias permitem o desenvolvimento de um software conforme os padrões recomendados pelo W3C.

Inicialmente, fez-se a restauração do arquivo que continha a aplicação e encontrava-se no servidor de aplicações web, gerando os vários arquivos que compunham o sistema. Foram identificados os arquivos das bibliotecas nativas da linguagem, os arquivos inerentes a projetos elaborados com o NetBeans e os arquivos próprios do MORE. Desta forma pode-se identificar e separar os códigos existentes por suas devidas linguagens, tais como: Java, HTML, CSS, Java Script e SQL.

$\mathrm{Na}$ sequência, procedeu-se à engenharia reversa ${ }^{2}$ do sistema, possibilitando a elaboração do Documento de Requisitos do MORE, onde todos os aspectos relativos às suas funcionalidades e possibilidades são especificados e modelados. Destaca-se que o referido documento aborda os seguintes itens: Concepção do Sistema; Visão Geral do Produto; Premissas, Restrições e Dependências; Arquitetura do Sistema; Requisitos Funcionais; Requisitos Não Funcionais; Modelagem dos Casos de Uso; Modelagem Lógica do Banco de Dados; Diagrama de Sequência e Comunicação; e, Diagrama de Estado.

A fim de esclarecer e exemplificar sobre a visão sistêmica que se teve ao realizar este trabalho, na Figura 2 mostra-se o Diagrama de Estado para Cadastro de Referências a Livros.

\footnotetext{
${ }^{2}$ Segundo Serra (2009), engenharia reversa "é um processo retroativo do ciclo de vida, que parte de um baixo nível de abstração (código-fonte ou executável) para um alto nível de abstração (modelos de projeto e especificação)".
} 
Figura 2: Diagrama de Estado para Cadastro de Referências a Livros

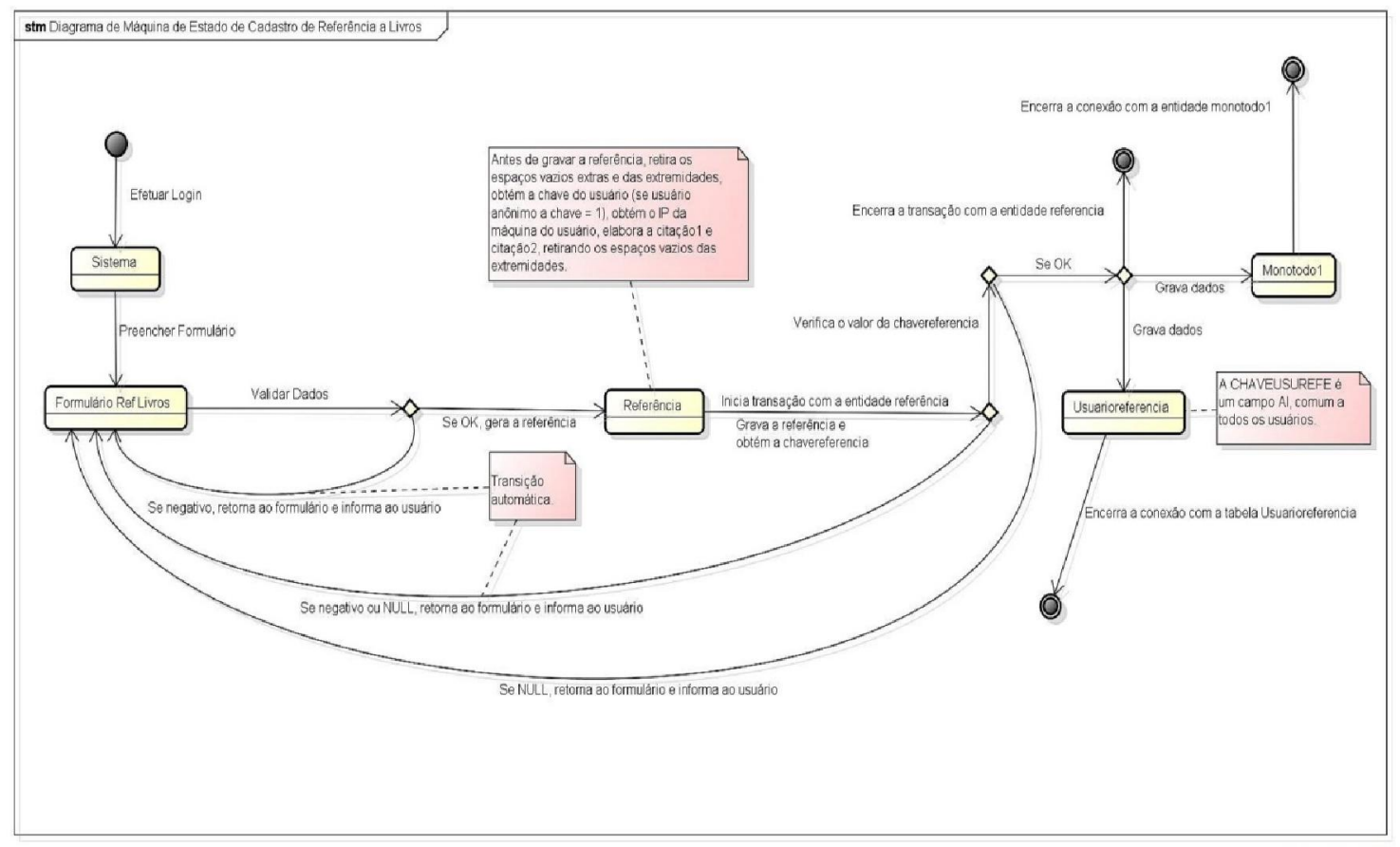

Fonte: Elaborada pelos autores.

A melhoria da estrutura do MORE deu-se basicamente em função da utilização do framework CodeIgniter que provê a estruturação do sistema em três camadas distintas e facilmente identificáveis. Este padrão de design é conhecido como MVC, que segundo Gabardo (2012), é "uma solução para desacoplar as camadas de lógica e regras de negócio da camada de apresentação. Implementa três camadas distintas, sendo elas Model, View e Controller, cada qual com suas características e atribuições".

Salienta-se que as funcionalidades afins foram divididas, inicialmente, em três grupos (módulos), a saber: suporte, controle de usuários e controle de referências. A estrutura dos dados sofreu poucas alterações para adequarse a nova conformação do sistema, uma vez que os dados existentes continuam sendo utilizados e não houve migração de banco de dados.

\section{ATUALIZAÇÃO DO MORE}

A atualização aconteceu de forma iterativa e incremental seguindo os preceitos de metodologias ágeis, mais precisamente o SCRUM que é calcado em sprints, ou seja, durante o ciclo de desenvolvimento faz-se a previsão de entregas (releases) que requerem a inclusão de novas funcionalidades devidamente testadas.

A utilização de tecnologias gratuitas foi respeitada durante todo o processo de modernização. Os modelos foram desenvolvidos no Astah Communit e o projeto do código fonte no Aptana Studio 3. 0 gerenciador dos dados continua sendo o SGDBR MySQL. As linguagens utilizadas são as recomendadas pelos principais desenvolvedores mundiais e aprovadas pelo W3C (World Wide Web Consortium) que é o principal órgão de padronização da web. São elas: HTML5, CSS3, JavaScript e PHP. Igualmente gratuitos são os 
frameworks utilizados, o CodeIgniter e o Zurb Foundation. Destaca-se que algumas rotinas do Zurb Foundation são implementadas com o uso do jQuery.

Buscou-se neste trabalho preservar as características antigas e atender às sugestões e críticas recebidas dos usuários durante estes oito anos à disposição da comunidade em geral. Na primeira entrega foi construído o módulo de suporte com as seguintes funcionalidades: Início (é a página inicial), Pesquisar (o usuário fornece uma cadeia de caracteres, no mínimo 5 caracteres, e o sistema retorna uma lista de referências que contenha aquela sequência), Ajuda (disponibiliza informações úteis para o preenchimento dos campos dos formulários), Sobre o MORE (informações sobre o contexto em que o MORE foi desenvolvido e a composição da equipe de desenvolvimento), Links (relação de links relacionados ao tema), Contato (formulário para o usuário comunicar-se com a equipe do MORE), Tutorial (é uma espécie de passo a passo para operar o sistema) e FAQ (lista de perguntas mais comuns com as respectivas respostas).

Posteriormente, o módulo do usuário foi desenvolvido, testado e integrado ao módulo de suporte. Nesse módulo encontram-se as funções que requerem algum controle mais apurado. Encontra-se no módulo do usuário, no link "Minha Conta": Cadastrar Usuário (formulário para cadastro de novos usuários), Atualizar Dados Usuário (permite ao usuário efetuar alguma correção no seu cadastro), Criar e Editar Coleções (possibilita organizar as referências geradas segundo algum critério pessoal e/ou projeto), Gerenciar Referências (possibilita a edição ou exclusão lógica de uma referência). Também está implantado o fornecimento automático de senha e login para quem esquecê-los e vier a solicitá-los, através de link localizado no formulário de identificação do usuário no sistema. 0 processo de identificação utiliza-se de sessões e não de cookies.

Nas demais entregas, referentes ao módulo de controle de referências, foram implementados os formulários para a geração de referências bibliográficas e citações abrangendo quase todos os tipos de fontes previstas na NBR 6023/2002.

A Figura 3 mostra a tela de início do sistema em operação. 
Figura 3: Tela de início do MORE

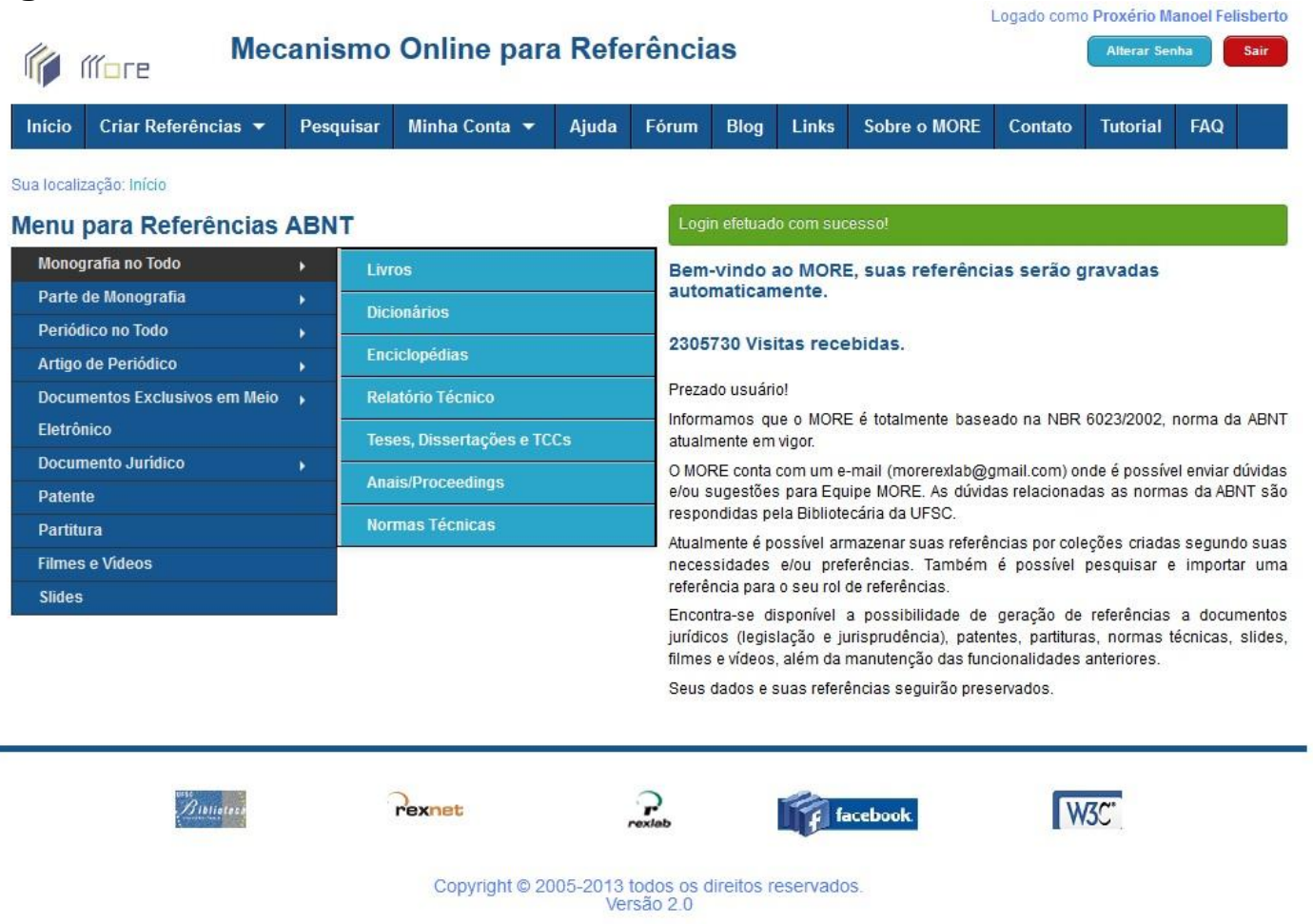

Fonte: print screen da aplicação em execução no navegador Mozilla Firefox.

O servidor web utilizado é o Apache e o MORE encontra-se, desde o dia 19 de outubro de 2013, em produção no RExLab da UFSC, Campus Araranguá, e seu acesso está disponível através da URL $<$ http://www.more.ufsc.br>.

\section{CONSIDERAÇÕES FINAIS}

A decisão tomada no sentido de submeter o MORE a um processo de atualização foi fruto de um período de acompanhamento do seu desempenho (principalmente através dos dados fornecidos pelo Google Analytics), dos feedbacks e sugestões dos usuários, além de minuciosa análise por parte da equipe envolvida no projeto.

Hoje, sabe-se que a decisão outrora tomada e levada a efeito com a execução do projeto dela derivado, vem dando resultados que podem ser verificados através de uma breve comparação entre alguns dados monitorados (médias) antes e depois da atualização. Em julho de 2012 ocorriam 1250 visitas diárias, o usuário permanecia no sistema por volta de cinco minutos e cinquenta e cinco segundos, percorria 2,11 páginas, com uma taxa de rejeição de 59,61\%. Em outubro do corrente ano, verifica-se um incremento de mais de 25.000 novos cadastros de usuários e que ocorrem em torno de 4.550 visitas diárias. 0 usuário, atualmente, permanece no sistema por volta de vinte e três minutos e quarenta e oito segundos, percorre 6,57 páginas, com uma taxa de rejeição de $18,48 \%$. No último mês foram recebidas visitas de hosts localizados em 58 países diferentes (perfazendo 3794 redes distintas) e redirecionamentos de 371 locais, destacando-se o Google com mais de $50 \%$ dos redirecionamentos recebidos, 
uma vez que o sistema encontra-se posicionado no início da primeira página de resultados, para determinados termos de pesquisa.

Desta forma, o Mecanismo Online para Referências (MORE) é apresentado como uma opção ao usuário de biblioteca, que necessita gerar e gerenciar suas referências bibliográficas, mas que não deseja despender recursos monetários, porém, busca uma ferramenta web, em português, que gere estas referências segundo as normas da ABNT, que ofereça a possibilidade de armazená-las e que esteja à sua disposição a qualquer hora.

Salienta-se que a equipe segue trabalhando no sentido de melhorar a percepção de utilidade e satisfação do usuário, através da implementação de um motor de busca em sua base de dados para reduzir os tempos de resposta nas pesquisas às referências armazenadas, bem como, utilizar-se dos metadados dos identificadores digitais de objetos para preenchimento automático de formulários de geração de referências bibliográficas.

\section{REFERÊNCIAS}

ALVES, M. B. M.; MENDES, L. L.; ALVES, J. B. da M. More: Mecanismo on-line para Referências. 2007. Disponível em:

http:/ /xa.yimg.com/kq/groups/25169972/1250144979. Acesso em $14 / 09 / 2014$.

ASSOCIAÇÃO BRASILEIRA DE NORMAS TÉCNICAS (ABNT). Conheça a ABNT. 2014. Disponível em: http://www.abnt.org.br/m3.asp?cod_pagina=929. Acesso em 21/10/2014.

ASSOCIAÇÃO BRASILEIRA DE NORMAS TÉCNICAS (ABNT). NBR 6023: Informação e Documentação - Referências - Elaboração. Rio de Janeiro, ago. 2002. 24 p.

BLANKENSHIP, J.; BUSSA, M.; MILLETT, S. Pro Agile .NET Development with Scrum. New York, US: Apress Media, 2012. 381 p.

CERVO, A. L.; BERVIAN, P. A.; SILVA, R. da. Metodologia Científica. 6. ed. São Paulo: Pearson Prentice Hall, 2007. 159 p.

ESTORNIOLO FILHO, J. EndNote Web: guia de uso. 6. ed. São Paulo, jan. 2013. 29 p. Disponível em: http://www.bvs-

sp.fsp.usp.br:8080/image/pt/internas/manuais/endnoteweb.pdf. Acesso em 06/09/2014.

GABARDO, A. C. PHP e MVC com CodeIgniter. 1. ed. São Paulo: Novatec, 2012. $286 \mathrm{p}$.

GILMORE, W. J. Dominando PHP e MySQL: do iniciante ao profissional. 3. ed. Rio de Janeiro: Alta Books, 2011. 769 p. Tradução de Raquel Marques e Lúcia Kinoshita. 2a reimpressão. 
GONÇALVES, E. Dominando o Netbeans. Rio de Janeiro: Ciência Moderna, 2006.

HOLZNER, S. Eclipse. Sebastopol, US: O'Reilly Media, 2004. 317 p.

LOUDON, K. Desenvolvimento de Grandes Aplicações Web. São Paulo: Novatec Editora, 2010. Tradução: Rafael Zanolli.

PALOMAR COLLEGE LIBRARY. EasyBib: Write Smart. [S.l.], 2014. Disponível em: http://www.palomar.edu/library/easybib/what-is-EasyBib.pdf. Acesso em $17 / 10 / 2014$.

PESTANA, M. C.; FUNARO, V. M. de O. Manual EndNote Web: guia do usuário. São Paulo, 2009. 23 p. Disponível em:

http://143.107.23.244/sdo/arquivos/Manual_EndNote_Web.pdf. Acesso em 06/09/2014.

SERRA, A. P. G. Reengenharia de software: uma visão geral. Engenharia de Software Magazine. Rio de Janeiro, n. 11, p. 20-24, 2009. Mensal.

SILVA, M. S. CSS3: desenvolva aplicações web profissionais com uso dos poderosos recursos de estilização das CSS3. São Paulo: Novatec, 2012.

SILVA, M. S. JavaScript: guia do programador. São Paulo: Novatec, 2010. $604 \mathrm{p}$.

SOMMERVILLE, I. Engenharia de Software. 9. ed. São Paulo: Pearson Prentice Hall, 2011. 529 p. Tradução Ivan Bosnic e Kalinga G. de O. Gonçalves.

SOURCEFORGE.NET. JabRef Reference Manager: documentation. 2014. Disponível em: http://jabref.sourceforge.net/documentation.php. Acesso em 05/07/2014.

THE LANDMARK PROJECT. Citation Machine. [S.l.], 2014. Disponível em: http://citationmachine.net/index2.php. Acesso em 15/09/2014.

TONU, M. A. H. PHP Application Development with NetBeans: Beginner's guide. Birmingham, UK: Packt Publishing, 2012. 283 p.

UPTON, D. CodeIgniter for Rapid PHP Application Development. Birmingham, UK: Packt Publishing, 2007. 244 p.

UNIVERSIDAD DE SEVILLA. PROCITE: Gestión de referencias bibliografías. fev. 2006. Disponível em:

http://bib.us.es/aprendizaje_investigacion/publicar_citar/herramientas/co mmon/procite.pdf. Acesso em 09/09/2014.

VAUGHAN-NICHOLS, S. J. Will HTML5 restandardize the web?. Computer, v. 43, n. 4, p. 13-15, April 2010. 